\title{
A Case of Yellow Nail Syndrome with Dramatically Improved Nail Discoloration by Oral Clarithromycin
}

\author{
Manabu Suzuki ${ }^{a-c}$ Atsuto Yoshizawa ${ }^{a}$ \\ Haruhito Sugiyama $^{a}$ Yasunori Ichimura ${ }^{a}$ Akane Morita ${ }^{a}$ \\ Jin Takasaki ${ }^{a}$ Gou Naka ${ }^{a}$ Satoshi Hirano ${ }^{a}$ Shinyu Izumi ${ }^{a}$ \\ Yuichiro Takeda ${ }^{a}$ Masayuki Hoji ${ }^{a}$ Nobuyuki Kobayashi ${ }^{a}$ \\ Koichiro Kudo ${ }^{a}$ \\ ${ }^{a}$ Department of Respiratory Medicine, National Center for Global Health and \\ Medicine, and ${ }^{\mathrm{b}}$ Internal Medicine, Department of Pulmonary Medicine, \\ Infection, Oncology, Nippon Medical School, Tokyo, and ${ }^{\mathrm{C} B i o s c i e n c e ~ M e d i c a l ~}$ \\ Research Center, Niigata University Medical and Dental Hospital, Niigata, Japan
}

\section{Key Words}

Nail discoloration $\cdot$ Yellow nail syndrome $\cdot$ Sinobronchial syndrome $\cdot$ Clarithromycin

\begin{abstract}
An 80-year-old woman was admitted to our hospital with pneumonia and exacerbation of sinobronchial syndrome (SBS). She presented with yellow discoloration of the nail beds of all fingers and toes, and her nails were recognized as growing slowly. Chest X-ray revealed bronchiectasis in the bilateral lower lobe and bilateral pleural effusion. We diagnosed her as having yellow nail syndrome (YNS), based on the triad of yellow nails, lymphedema, and lung disease. After treatment with antibiotics [ampicillin/sulbactam and clarithromycin (CAM)] for pneumonia and SBS, her general condition improved, and the yellow nails disappeared in some fingers. When she was previously treated with $200 \mathrm{mg}$ CAM for SBS, her yellow nails had not shown improvement. This time, her yellow nails improved after treatment with 400 mg CAM. The literature reports vitamin E, zinc, and topical corticosteroid plus active vitamin D3 to be effective in the treatment of yellow nails. Two studies have reported treatment for YNS using CAM, though they found a lack of efficacy. Thus, the present case is the first to report improved yellow nails using CAM alone. We conclude that not only SBS and lung disease but also YNS were improved by treatment with 400 mg CAM.
\end{abstract}




\section{Introduction}

Yellow nail syndrome (YNS) is a rare disorder of unknown cause characterized by the triad of yellow discoloration and thickened nails with slow growth, chronic lymphedema, and respiratory manifestations, such as bronchiectasis or pleural effusion $[1,2]$. We examined a patient with YNS and underlying sinusitis, who suffered from slow growth and yellow discoloration of the nails, bronchiectasis, bilateral pleural effusion, and peripheral lymphedema. Based on the presence of the three basic symptoms, the patient was diagnosed as having YNS. We report the efficacy of clarithromycin (CAM) for treating yellow nails: only $400 \mathrm{mg}$ per day dramatically improved the yellow nail discoloration and growth of nails in this patient.

\section{Case Report}

An 80-year-old woman was admitted to our hospital because of pneumonia. Her symptoms were loss of appetite, pyrexia, and purulent sputum, which had worsened despite CAM treatment of $200 \mathrm{mg}$ per day. The underlying diseases were a colon polyp, sinusitis, and Candida onychomycosis infection. On physical examination, her height was $145 \mathrm{~cm}$, her weight $35 \mathrm{~kg}$, blood pressure 108/60 mm Hg, pulse rate 70 beats $/ \mathrm{min}$ and regular, body temperature $36.6^{\circ} \mathrm{C}$, and respiratory rate 21 per minute. The patient was lucid, and she did not suffer from anemia or icterus; the superficial lymph nodes were not swollen. On auscultation, the breath sounds revealed a coarse crackle in the bilateral lower fields. The abdomen was soft and flat. Neurological examination results were normal. There was a slight peripheral pitting edema at the ankle joints. Bilaterally, the nails of the fingers and toes were of a yellow-green color; there were thickness of the beds, onycholysis, and slow growth, as a result of which she had not cut her nails for over 6 months. The blood test showed an increasing inflammatory reaction and slight hypoxia (table 1). Urinary findings, renal function, and brain natriuretic peptide values were normal. Deep mycosis was also considered because of her nail discoloration, but beta-Dglucan and candida antigen tests were negative. The initial X-ray revealed infiltration in the bilateral lower fields, in particular a lack of cardiac silhouette over a part of the left diaphragm, 'tramline' shadows as a clinical feature of bronchiectasis, and blunting of the bilateral costophrenic angles (fig, 1).

The patient was diagnosed as having YNS because her nails had previously been resistant to therapy for nail candidiasis and she had lung complications. After admission, she was treated with $4.5 \mathrm{~g}$ per day of sulbactam/ampicillin for pneumonia and $400 \mathrm{mg}$ per day of CAM for exacerbation of sinobronchial syndrome (SBS). Since her respiratory tract symptoms and inflammatory changes were showing improvement after 10 days of treatment, the nail discoloration and slow growth were recognized as having been ameliorated by this treatment.

The patient had previously been diagnosed with nail candidiasis, but the nails did not improve despite mycosis treatment. Fig. 2 shows the clinical course of treatment. Since 2001, the patient has been treated with a topical antifungal cream for her nails. Since 2002, she has also received $200 \mathrm{mg}$ CAM per day as SBS treatment. Though CAM treatment was discontinued following an improvement in the patient's lung complaints, the nail lesions were unaffected. Subsequently, the nail improvement was achieved following treatment with $400 \mathrm{mg}$ CAM daily.

Photographs of the nails before and after treatment are shown in fig. 3 . After the treatment, the yellow-green areas of the nails had decreased at the nail matrix, and at the nail matrix new nail growth was found. In particular, the small finger showed great improvement in nail discoloration and slow growth; subsequently, her nails required cutting. The patient was administered only with intravenous antibiotics and oral macrolide. We consider that CAM treatment was more effective for YNS.

The patient continues to receive outpatient treatment of $400 \mathrm{mg}$ CAM per day, and the condition of her nails is improving. 


\section{Discussion}

YNS is a rare disorder of unknown cause characterized by the classic triad of yellow and thickened nails, lymphedema, and respiratory manifestations. In 1964, Samman and White [1] described YNS in 13 cases. Typically, the nails grow slowly, are excessively curved, and show a yellow discoloration. They frequently show ridging owing to interrupted growth. Onycholysis can occur in one or more nails. On the other hand, in 1966 Emerson [2] described 3 patients with yellow discoloration of the nails, pleural effusion, and chronic lymphedema as representing a new syndrome. The patient in the present study had slow-growing and yellow nails, lymphedema, and respiratory manifestation, and she was confirmed to have YNS.

According to an analysis of 97 YNS patients reported by Nordkild et al. [3], 89\% had yellow nails, $80 \%$ had lymphedema, and $36 \%$ had lung disease. Of 50 cases reported by Mambretti-Zumwalt et al. [4], only 13 (27\%) had the complete triad of symptoms. Though visible change in the peripheral edema did not appear in the present case, yellow nails and bronchiectasis with pleural effusion led to the diagnosis of YNS.

In the differential diagnosis, nail inspection takes into account focal infections, such as nail candidiasis and tinea unguium. Such cases can be confirmed by mycological examination based on fungal cultures of the nails or microscopic visualization of the fungal forms. Changes in the condition of the nails and response to therapy vary with time. In a refractory case where all finger- or toenails are discolored and recognized as being associated with respiratory disease, the diagnosis should be YNS.

The cause of YNS is unknown. It has been suggested as being a congenital lymph abnormality, though one in which the disorder is very mild. In addition to skin or respiratory infection, any stress, such as trauma, after growth leads to obvious lymphatic drainage abnormalities and results in lymphedema. These changes produce an overall rate of growth that is usually less than one tenth that of normal [1]. The nail grows more slowly than $0.2 \mathrm{~mm} /$ week compared with the minimum of $0.5-1.2$ $\mathrm{mm} /$ week in healthy subjects. Because of the slow growth, the nails' color ranges from pale yellow or slightly greenish to shades of black, and the nails are thickened and usually excessively curved from side to side; onycholysis, disappearance of the lunulae, deficient cuticles, paronychia, and transversal grooves may be present [2].

The bronchiectasis that accompanies YNS often coexists with chronic sinusitis. Chronic sinusitis has been reported in 20-25\% cases of YNS [5]. The cause of bronchiectasis is also unknown, but it has been hypothesized that bronchiectasis may be induced by sinobronchial lymph duct occlusion [3]. Bronchial mucosa biopsy has demonstrated that edematous changes are associated with lymphatic congestion [6]. Therefore, we hypothesize that a recurrent respiratory tract infection caused by lymphatic congestion leads to a locally acquired immunocompromised state and bronchiectasis.

Vitamin E [7], zinc [8], and topical corticosteroid plus active vitamin D3 [3] have been reported to be effective for treating yellow nails. Two cases of treating YNS with CAM have been described. In one case of complicated SBS, respiratory disease and nail discoloration showed improvement following treatment with CAM and topical corticosteroid [5]. In the other case of complicated SBS associated with eosinophilic 
bronchial disease, the respiratory disease evidenced improvement, though the nail discoloration failed to improve following treatment with CAM and budesonide [9].

We will now consider the mechanisms whereby the nail disease improved following amelioration of the respiratory disease. It has been noted that 14-membered macrolide antibiotics, including CAM, have various anti-inflammatory effects in addition to the antibacterial effects. They inhibit water and mucus secretion by the tracheal epithelium, chloride secretion across canine tracheal epithelial cells [10], and MUC5AC production [11]. These antibiotics also inhibit inflammatory cytokines and the antiproliferative effect of inflammatory cells, such as decreasing secretion of IL-6 and GMCSF from epithelial cells, inhibiting IL-2, -3 , and -4 production from lymphocytes, and decreasing the production of IL-1, TNF $\alpha$, and IL-8 from monocytes and macrophages [12]. In our patient, the nail improvement appeared to be associated with better control of the respiratory manifestations following treatment of SBS with CAM. In fact, several authors have reported some improvement in the nail abnormalities following better control of the respiratory manifestations or decongestive therapy for lymphedema. We examined the relationship between dose and effect of CAM in our patient. When she was treated with $200 \mathrm{mg}$ CAM for SBS in 2002, there was no improvement in her yellow nails. At that time, improvement in the nail coloration occurred after treatment with an increased dose of $400 \mathrm{mg}$ CAM. Ianaro et al. [13] reported the anti-inflammatory activity of macrolide antibiotics. In the present study, we described how both the exudate volume and leukocyte accumulation were dose-dependently reduced by CAM. Furthermore, the production of IL-1 $\beta$, TNF- $\alpha$, and IL- 6 were also reportedly reduced by macrolides [13]. A dose of over $400 \mathrm{mg}$ CAM has the potential to improve the nail discoloration in YNS.

The literature reports no evidence of nail improvement in YNS using CAM alone. Thus, the present case is the first report of such yellow nail improvement using only CAM. Treatment with over $400 \mathrm{mg}$ CAM for YNS accompanied with sinusitis may improve not only the sinusitis and lung disease but also the yellow nails associated with lymphedema of the periungual tissue. 
Table 1. Laboratory data on admission

\begin{tabular}{|c|c|c|c|c|c|c|c|}
\hline \multicolumn{2}{|c|}{ Hematology } & \multicolumn{2}{|c|}{ Serology } & \multicolumn{2}{|c|}{ Immune serum } & \multicolumn{2}{|c|}{ Atrial blood gas } \\
\hline WBC & $10,560 / \mu \mathrm{l}$ & Alb & $2.8 \mathrm{~g} / \mathrm{dl}$ & $\beta$-D glucan & $6.6 \mathrm{pg} / \mathrm{ml}$ & $\mathrm{pH}$ & 7.369 \\
\hline Neu & $87.6 \%$ & AST & $43 \mathrm{U} / 1$ & $\mathrm{BNP}$ & $5.9 \mathrm{pg} / \mathrm{ml}$ & $\mathrm{PCO}_{2}$ & $38.3 \mathrm{~mm} \mathrm{Hg}$ \\
\hline Lym & $8.3 \%$ & ALT & $28 \mathrm{U} / \mathrm{l}$ & $\operatorname{IgG}$ & $1,744.7 \mathrm{pg} / \mathrm{dl}$ & $\mathrm{PO}_{2}$ & $69.5 \mathrm{~mm} \mathrm{Hg}$ \\
\hline Mon & $4.2 \%$ & LDH & $211 \mathrm{U} / \mathrm{l}$ & $\operatorname{IgA}$ & $169.8 \mathrm{pg} / \mathrm{dl}$ & $\mathrm{HCO}_{3}$ & $23.5 \mathrm{mmol} / \mathrm{l}$ \\
\hline Eos & $0.1 \%$ & $\gamma \mathrm{GTP}$ & $23 \mathrm{U} / \mathrm{l}$ & IgM & $46.3 \mathrm{pg} / \mathrm{dl}$ & & \\
\hline Bas & $0.1 \%$ & BUN & $13.8 \mathrm{mg} / \mathrm{dl}$ & T-IgE & $15.0 \mathrm{U} / \mathrm{ml}$ & & \\
\hline $\mathrm{RBC}$ & $399 \times 10^{4} / \mu \mathrm{l}$ & Cre & $0.52 \mathrm{mg} / \mathrm{dl}$ & & & & \\
\hline $\mathrm{Ht}$ & $33.1 \%$ & $\mathrm{Na}$ & $135 \mathrm{mEq} / \mathrm{l}$ & & & & \\
\hline $\mathrm{Hb}$ & $10.0 \mathrm{~g} / \mathrm{dl}$ & $\mathrm{K}$ & $3.6 \mathrm{mEq} / \mathrm{l}$ & & & & \\
\hline \multirow[t]{2}{*}{ Plt } & $28.4 \times 10^{4} / \mu \mathrm{l}$ & $\mathrm{Cl}$ & $99 \mathrm{mEq} / \mathrm{l}$ & & & & \\
\hline & & CRP & $18.01 \mathrm{mg} / \mathrm{dl}$ & & & & \\
\hline
\end{tabular}

WBC = White blood cell; Neu = neutrophil; Lym = lymphocyte; Mon = monocyte; Eos = eo-sinophil; Bas = basophil; $\mathrm{RBC}=$ red blood cell; $\mathrm{Hb}=$ hemoglobin; $\mathrm{Ht}=$ hematocrit; $\mathrm{Plt}=$ platelet; $\mathrm{Alb}=$ albumin; $\mathrm{AST}=$ aspartate aminotransferase; $\mathrm{ALT}=$ alanine aminotransferase; $\mathrm{LDH}=$ lactate dehydrogenase; $\gamma \mathrm{GTP}=\gamma$ glutamyl transpeptidase; $\mathrm{BUN}=$ blood urea nitrogen; $\mathrm{Cr}=$ creatinine; $\mathrm{Na}=$ sodium; $\mathrm{K}=$ potassium; $\mathrm{Cl}=$ chloride; $\mathrm{CRP}=\mathrm{C}$-reactive protein; $\mathrm{BNP}=$ brain natriuretic peptide; IgG = immunoglobulin $\mathrm{G}$; $\operatorname{IgA}=$ immunoglobulin A; IgM = immunoglobulin $\mathrm{M}$; T-IgE = total immunoglobulin $\mathrm{E} ; \mathrm{HCO}_{3}=$ bicarbonate ion; $\mathrm{PCO}_{2}=$ partial pressure of arterial carbon dioxide; $\mathrm{PO}_{2}=$ partial pressure of arterial oxygen .

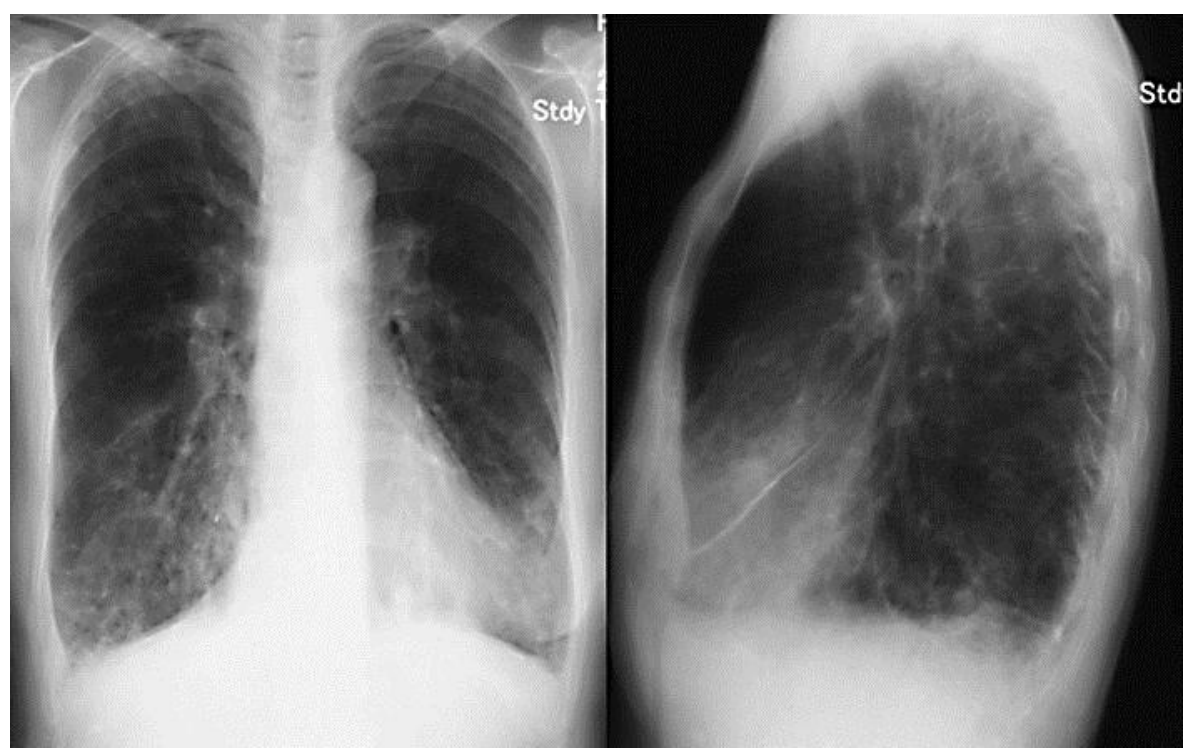

Fig. 1. Chest X-ray on admission showed bilateral lower lobe infiltration. 'Tramline' revealed in the right lower lobe. 
Figure 2 Clinical course

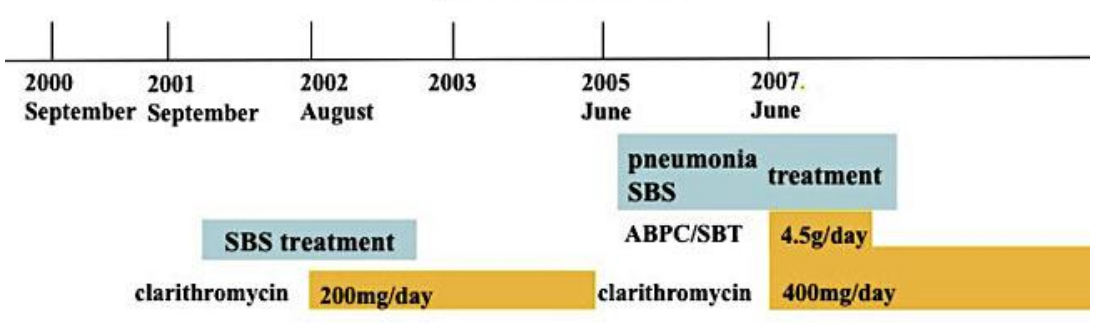

\section{Nail candidiasis treatment}

butenafine once/day

bifonazole once/day

terbinafine $125 \mathrm{mg} /$ day

glycerin $600 \mathrm{mg} / \mathrm{day}$

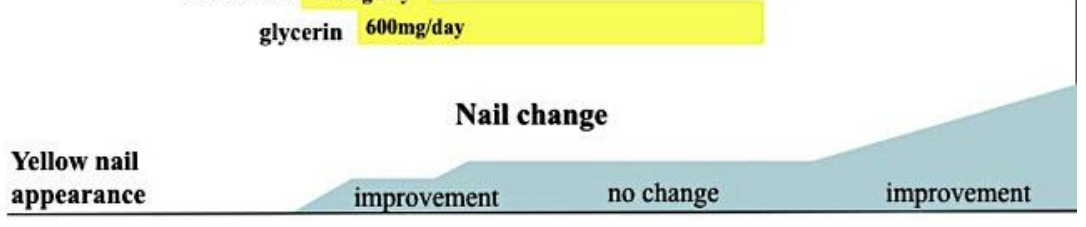

Fig. 2. Clinical history of the patient with regard to nail discoloration and sinobronchial syndrome (SBS). 

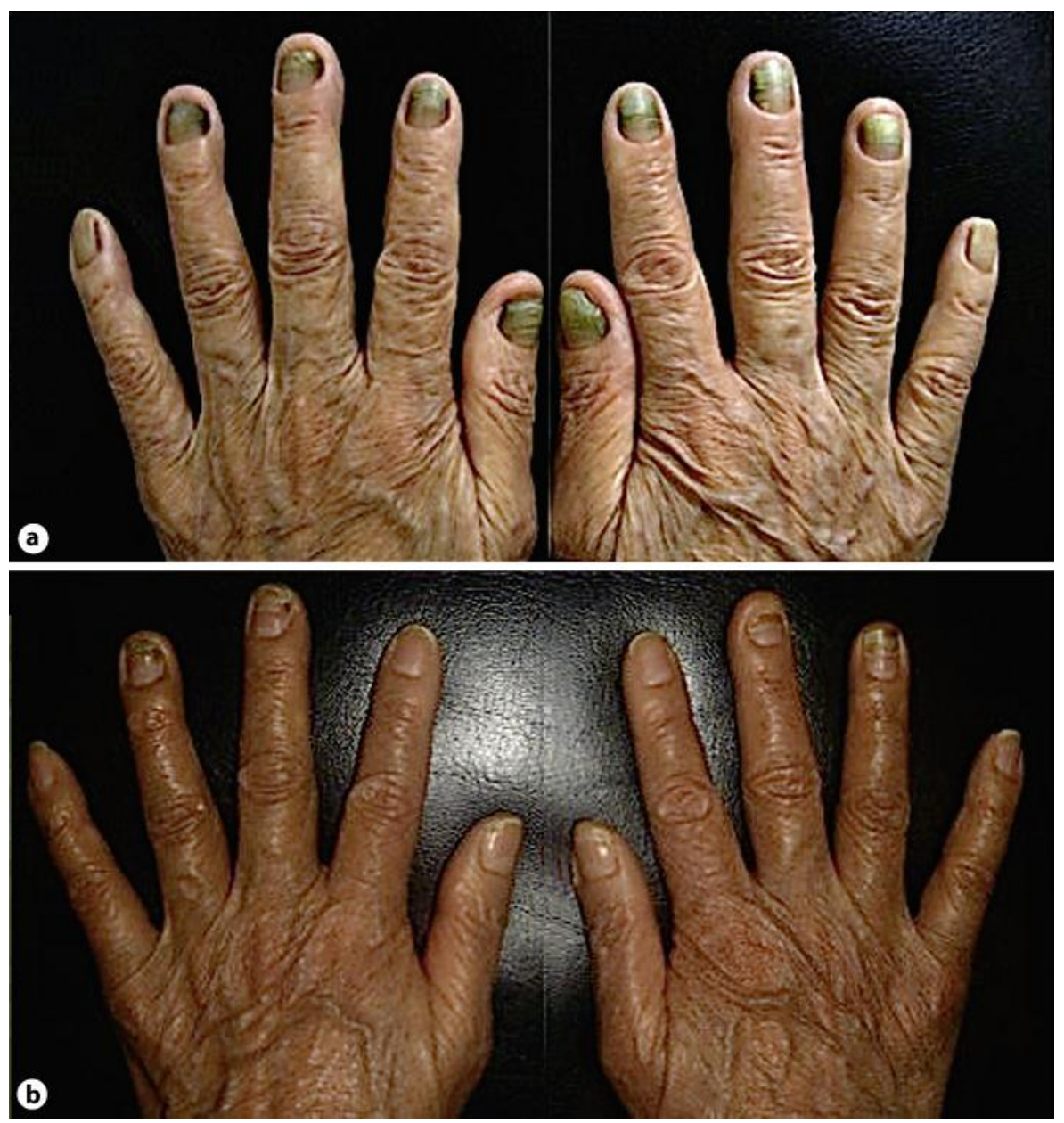

Fig. 3. The yellow nails improved dramatically after 9 months of CAM treatment. a Pretreatment. b Posttreatment.

\section{References}

1 Samman PD, White WF: The yellow nail syndrome. Br J Dermatol 1964;76:153-157.

-2 Emerson PA: Yellow nail, lymphoedema and pleural effusion. Thorax 1966;21:247-253.

-3 Nordkild P, Kromann-Andersen H, Struve-Christensen E: Yellow nail syndrome - the triad of yellow nails, lymphedema and pleural effusions. A review of the literature and a case report. Acta Med Scand 1986;219:221-227.

4 Mambretti-Zumwalt J, Seidman JM, Higano N: Yellow nail syndrome: complete triad with pleural protein turnover studies. South Med J 1980;73:995-997.

5 Doi R, Okamoto H, Horio T: Yellow nail syndrome with major triad and sinusitis treated with clarithromycin (in Japanese). Jpn J Dermatol 2002;112:261-265.

-6 Tanoue M, Satoh T, Yoshizawa Y: A case of yellow nail syndrome presenting as recurrent pneumonia (in Japanese). Nihon Kyobu Shikkan Gakkai Zasshi 1993;31:1566-1571.

7 Ayres S Jr, Mihan R: Yellow nail syndrome: response to vitamin E. Arch Dermatol 1973;108:267-268.

-8 Arroyo JF, Cohen ML: Improvement of yellow nail syndrome with oral zinc supplementation. Clin Exp Dermatol 1993;18:62-64. 
9 Toyoshima M, Chinda K, Suda T: A case of yellow nail syndrome associated with eosinophilic bronchial disease successfully treated with clarithromycin and budesonide (in Japanese). Nihon Kokyuki Gakkai Zasshi 2005;43:508-512.

10 Tamaoki J, Isono K, Sakai N, Kanemura T, Konno K: Erythromycin inhibits Cl secretion across canine tracheal epithelial cells. Eur Respir J 1992;5:234-238.

11 Ikeda $\mathrm{K}, \mathrm{Wu} \mathrm{D}$, Takasaka T: Inhibition of acetylcholine-evoked $\mathrm{Cl}$ - currents by 14-membered macrolide antibiotics in isolated acinar cells of the guinea pig nasal gland. Am J Respir Cell Mol Biol 1995;13:449454

12 Morikawa K, Oseko F, Morikawa S, Iwamoto K: Immunomodulatory effects of three macrolides, midecamycin acetate, josamycin, and clarithromycin, on human T-lymphocyte function in vitro. Antimicrob Agents Chemother 1994;38:2643-2647.

13 Ianaro A, Ialenti A, Maffia P, Sautebin L, Rombola L, Carnuccio R, Iuvone T, D’Acquisto F, Di Rosa M: Antiinflammatory activity of macrolide antibiotics. J Pharmacol Exp Ther 2000;292:156-163. 